\title{
Magnetisation transfer ratio analysis of normal appearing white matter in patients with familial and sporadic multiple sclerosis
}

\author{
M Siger-Zajdel, K Selmaj
}

\begin{abstract}
Objectives-To assess differences in magnetisation transfer ratio (MTR) analysis of normal appearing white matter (NAWM) in patients with familial multiple sclerosis (MS) and those with sporadic MS.

Methods-10 patients with familial MS, 10 patients with sporadic MS, and 10 healthy subjects were included in the study. Groups were matched according to the sex, age, disease duration, type of disease, EDSS, and MRI T1 and T2 lesion load. Magnetisation transfer imaging (MTI) with and without saturation pulse were performed. On the MTR map 16 different regions of interest of normal appearing white matter were analyzed.

Results-The mean MTR value of normal appearing white matter was significantly lower both in familial patients and those with sporadic MS compared with healthy subjects $(33.8 \%$ v $46.4 \% ; 38.6 \%$ v $46.4 \%$ respectively, $p<0.05$ ). Additionally, patients with familial MS showed significantly lower mean MTR value than patients with sporadic MS $(33.8 \% v 38.6 \%$, $\mathbf{p}<0.05)$. There was also significant regional varation of MTR values between these two groups of patients.

Conclusions-Lower and more widespread MTR abnormalities in patients with familial MS might indicate differences in the extent and nature of white matter pathology between familial and sporadic MS.
\end{abstract}

( $F$ Neurol Neurosurg Psychiatry 2001;71:752-756)

Key words: familial multiple sclerosis, magnetic resonance imaging, magnetisation transfer ratio

The incidence of familial multiple sclerosis (MS) has been estimated as between 3.6\%$20 \%$ in the general MS population..$^{1-5}$ Epidemiological data have shown that the risk of MS development in first degree relatives of patients is five to 40 times higher than in the general population. ${ }^{6-9}$ Additionally, Sadovnick et $a l^{10}$ in a population based study estimated that MS would develop in $2 \%-3 \%$ of non-twin siblings and $3 \%-5 \%$ of children of patients with MS. Moreover, the incidence of MS has been shown to be $2 \%-5 \%$ and $25 \%-30 \%$ in dizygotic and monozygotic twins, respectively. ${ }^{11-12}$ This suggests that multiple genetic factors influence the occurence of MS. ${ }^{13-14}$ On the other hand environmental factors have also been considered in MS predispositions. ${ }^{15-16}$ Although demographic and clinical differences between patients with familial and those with sporadic MS are not significant there is still ongoing discussion on the distinction between these two forms of the disease. ${ }^{17-18}$

Magnetic resonance imaging is the most sensitive paraclinical tool to demostrate focal brain and spinal cord abnormalities in MS. ${ }^{19}$ However, several necropsy studies disclosed more widespread white matter pathology, including microscopical demyelination and cellular infiltration, which were not detectable on conventional MRI. These findings suggested that pathology in MS in not restricted to the focal lesions but extended to the adjacent white matter. ${ }^{20-22}$ To detect this subtle, microscopical white matter pathology in MS, magnetisation transfer imaging (MTI) can be applied. This is based on chemical exchange and cross relaxation between two pools of protons (bound and free protons). ${ }^{23-24}$ This transfer can be quantified as a magnetisation transfer ratio (MTR), which indirectly indicates destruction of the macromolecular matrix. ${ }^{25}$

There are a few reports concerning conventional MRI in patients with familial $\mathrm{MS}^{26-28}$ and there are no data on MTR comparison between patients with familial MS and patients with sporadic MS. In this study we have compared mean MTR and regional MTR values in normal appearing white matter in patients with these two forms of the disease.

\section{Patients and methods}

Patients with familial MS were defined as probands with one or more relatives having MS within one family (multiplex families). They were selected from clinical records of the Department of Neurology. All available records were analysed and MS probands and their affected relatives were included in the study. For final analysis only patients with complete clinical history and with clinically definite MS according to the Poser criteria ${ }^{29}$ were included. Ten patients with MS from 10 multiplex families with MS (five women and five men, six with a relapsing-remitting and four with a secondary progressive course of disease) and 10 patients with sporadic MS (five women and five men, six with a relapsing-remitting, four with a secondary progressive course) were included in the study. All patients were examined neurologically and their clinical status was measured using the expanded disability status scale (EDSS). To avoid any effect of demographic and clinical characteristics on MTR results, both MS groups, familial and sporadic, were adequately matched. None of the patients had 
Table 1 Clinical and conventional MRI results in familial and sporadic MS patients

\begin{tabular}{llll}
\hline & $\begin{array}{l}\text { Familial MS } \\
\text { patients }\end{array}$ & $\begin{array}{l}\text { Sporadic MS } \\
\text { patients }\end{array}$ & Control \\
\hline No of patients & 10 & 10 & 10 \\
Male/female ratio & 1 & 1 & 1 \\
Mean age (SD) (y) & $35.6(4.30)$ & $33.7(2.91)$ & $33.5(3.37)$ \\
Mean duration of the disease (SD) (y) & $6(1.2)$ & $5(2.1)$ & \\
Mean EDSS (SD) & $4.0(1.33)$ & $4.0(0.91)$ & \\
Mean T1 lesion load $\left(\mathrm{mm}^{3}\right)$ & $230.0(111.21)$ & $310.1(21.34)$ & \\
Mean T2 lesion load $\left(\mathrm{mm}^{3}\right)$ & $2662.40(93.78)$ & $2569.30(47.61)$ & \\
\hline
\end{tabular}

had relapses or steroid treatment during the 5 months preceding study entry. Additionally, they had neither immunosupressive nor immunomodulative treatment during the 6 months before the study. Both groups were also standardised according to $\mathrm{T} 1$ and $\mathrm{T} 2$ image burden (table1). Additionally, 10 healthy subjects age and sex matched with the patients entered the study as a control group. All subjects gave written, informed consent before entering the study, which had been approved by the local ethics commitee.

\section{IMAGE ACQUISITION}

All examinations were performed on the same 1.5 T MR system (Vision, Siemens, Erlangen, Germany). In all patients the following sequences were acquired during a single session: (1) dual echo; repetition time (TR) $4.500 \mathrm{~ms}$; first echo time (TE) $22 \mathrm{~ms}$; second echo TE 90 $\mathrm{ms}$; section thickness $3 \mathrm{~mm}$, intersection gap $0.1 \mathrm{~mm}, 23$ slices, matrix $190 \times 256$; number of acquisitions and two dimensional gradient echo (GE; TR 800 ms; TE 10 ms; flip angle 30 degrees; slice thickness $3 \mathrm{~mm}$, intersection gap $0.1 \mathrm{~mm}, 23$ slices, matrix $192 \times 256$ ) with and without saturation pulse (off resonance RF pulse centred $1.5 \mathrm{kHz}$ below the water frequency, gaussian envelope duration $14.3 \mathrm{~ms}$, a band width $130 \mathrm{~Hz}$, and an amplitude of $3.13 \times 10^{-6} \mathrm{~T}$ ) were obtained. The slices in all sequences were positioned to run parallel to a line that joins the most inferoposterior parts of the corpus callosum according to published guidelines. ${ }^{30}$

IMAGE ANALYSIS

Firstly, macroscopic MS lesions were identified by a single observer (unaware of the identity of the patients) on T2 and T1 weighted images. $\mathrm{T} 1$ weighted lesions were defined as an area of signal intensity between that of the grey matter and that of the CSF, and with corresponding lesions on both echos of the dual echo images and sharply demarcated from surrounding tissue. Then T 2 and T1 weighted lesion segmentation were performed using a semiautomated segmentation technique based on a local thresholding technique. Lesion load was calculated by adding the areas of all lesions and multiplying by slice thickness

A magnetisation transfer ratio (MTR) map was calculated from the two GE images, with and without the saturation pulse, on a pixel by pixel basis from the formula:

$\mathrm{MTR}=(\mathrm{Mo}-\mathrm{Ms}) / \mathrm{Mo} \times 100$

where Ms represents signal intensity with the saturation pulse and Mo represents signal intensity without the saturation pulse. ${ }^{24}$

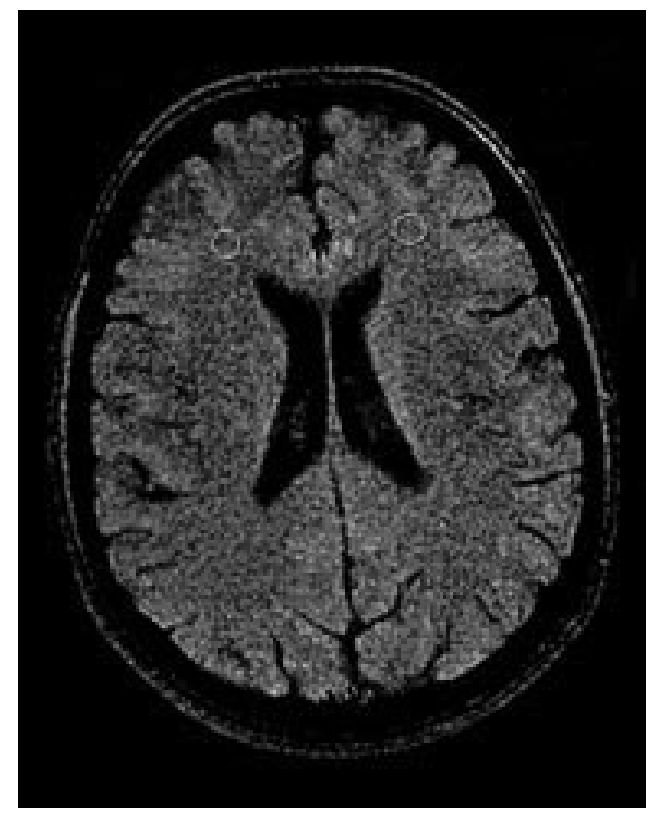

Figure 1 Image shows ROI measurements in normal appearing white matter on MTR map.

We performed region of interest (ROI) analysis of normal appearing white matter. Firstly we identified normal appearing white matter on T2 weighted images. The normal appearing white matter areas were those that were not surrounded on the same slices and in at least the two adjacent ones by MS lesions visible on the coregistered $\mathrm{T} 2$ weighted images as described before. ${ }^{31}$ Then the round cursor $\left(25 \mathrm{~mm}^{2}\right)$ was placed in 16 different areas: bilaterally in the centrum semiovale, frontal and occipital white matter, internal capsules, cerebellum, and cerebral and cerebellar peduncles and in the central portion of the genu and splenium of the corpus callosum (fig 1 ). If the ROI was located in a macroscopical lesion these data were not included in the final analysis. However, for the total 360 measurements in this study it occurred only twice. To avoid a partial volume effect from CSF and from grey matter the cursor was placed only in white matter and distant to CSF and grey matter. Additionally, adjacent slices were evaluated to ensure that ROIs did not include CSF or grey matter. Then signal intensity values for all 16 ROIs were measured on MTR map. For all participants, ROIs were identical in size and shape for each studied area. On the basis of 16 MTR values the mean MTR value for each participant was calculated. Additionally, using MTR values of each bilaterally situated ROI mean MTR values of these seven points were also calculated.

STATISTICAL ANALYSIS

Comparisons between different types of patients and the control group for MTR data were assessed using one way analysis of variance (ANOVA). For post hoc analysis, multiple comparison tests with Bonferroni correction were used. For detailed analysis of MTR ROI differences between each pair of subjects an additional multiple comparison test was used (Duncan's test). To compare clinical 
Table 2 Comparison of mean MTR values (SD) between all investigated groups

\begin{tabular}{llc}
\hline & & $p$ Value $^{\star}$ \\
\hline Familial MS patients & $v$ control group & \\
$33.8 \%(0.08)$ & $46.4 \%(0.06)$ & $<0.05$ \\
Sporadic MS patients & $v$ control group & \\
$38.6 \%(0.05)$ & $46.4 \%(0.06)$ & $<0.05$ \\
Familial MS patients & $v$ sporadic MS patients & \\
$33.8 \%(0.08)$ & $38.6 \%(0.05)$ & $<0.05$ \\
\hline${ }^{\star}$ p Value obtained from one way analysis of variance (ANOVA) & \\
with Bonferroni correction.
\end{tabular}

data and conventional MRI results the MannWhitney $U$ test was performed. Statistical significance was defined as $\mathrm{p}<0.05$.

\section{Results}

No white matter signal abnormalities were found in the control group. In MRI of all patients, white matter lesions fulfilled the MS critieria proposed by Barkhof et al. ${ }^{30}$ Both MS groups were matched for $\mathrm{T} 1$ and $\mathrm{T} 2$ lesion load ( $p>0.05$ for both indices; table 1$)$.

The mean MTR values of all analyzed groups are given in table 2 . Compared with the control group, the mean MTR obtained from patients with familial MS as well as from patients with sporadic MS was significantly lower $(\mathrm{p}<0.05)$. Interesingly, we also found significantly lower mean MTR values in patients with familial MS compared with sporadic patients $(\mathrm{p}<0.05)$.

Analysis of ROIs showed that in both familial and sporadic groups of patients mean MTR values in most analyzed regions were significantly lower than in the control group ( $\mathrm{p}<$ 0.05; tables 3 and 4). Additionally, mean MTR values in most of ROIs of the patients with familial MS were significantly lower than in the

Table 3 Mean MTR values (SD) in 16 regions of interest

\begin{tabular}{|c|c|c|c|}
\hline Location & $\begin{array}{l}\text { Familial MS } \\
\text { patients (\%) }\end{array}$ & $\begin{array}{l}\text { Sporadic MS } \\
\text { patients (\%) }\end{array}$ & Control group (\%) \\
\hline Centrum semiovale ${ }^{\star}$ & $34.1(0.20)$ & $32.1(0.12)$ & $47.9(0.11)$ \\
\hline Corpus callosum (genu) & $29.7(0.62)$ & $38.4(0.25)$ & $48.9(0.20)$ \\
\hline Corpus callosum (splenium) & $32.5(0.40)$ & $42.4(0.17)$ & $42.6(0.17)$ \\
\hline Frontal white matter ${ }^{\star}$ & $37.1(0.20)$ & $36.1(0.18)$ & $49.2(0.17)$ \\
\hline Occipital white matter ${ }^{\star}$ & $37.5(0.18)$ & $38.6(0.14)$ & $43.6(0.14)$ \\
\hline Internal capsule & $34.4(0.14)$ & $36.6(0.09)$ & $43.8(0.11)$ \\
\hline Cerebral peduncle ${ }^{\star}$ & $32.7(0.10)$ & $44.9(0.11)$ & $44.7(0.12)$ \\
\hline Cerebellar peduncle* & $33.5(0.17)$ & $41.1(0.12)$ & $50.0(0.11)$ \\
\hline Cerebellum ${ }^{\star}$ & $33.4(0.17)$ & $37.4(0.11)$ & $47.9(0.18)$ \\
\hline
\end{tabular}

^For the hemispheric regions, both right and left hemisphere values for MTR were included to the analysis.

Table 4 Significance ( $p$ values ${ }^{*}$ ) of ROI MTR differences between all investigated groups

\begin{tabular}{|c|c|c|c|}
\hline Location & $\begin{array}{l}\text { Familial MS v } \\
\text { control }\end{array}$ & $\begin{array}{l}\text { Sporadic MS v } \\
\text { control }\end{array}$ & $\begin{array}{l}\text { Familial v sporadic } \\
M S\end{array}$ \\
\hline Centrum semiovale† & $0.00014 \ddagger$ & 0.00006 & 0.00014 \\
\hline Corpus callosum (genu) & 0.00006 & 0.00014 & 0.00014 \\
\hline Corpus callosum (splenium) & 0.00006 & 0.10867 & 0.00014 \\
\hline Frontal white matter† & $0.00014 \ddagger$ & 0.00006 & 0.00014 \\
\hline Occipital white matter & 0.00006 & 0.00014 & 0.00014 \\
\hline Internal capsule $†$ & 0.00006 & 0.00014 & 0.00014 \\
\hline Cerebral peduncle & 0.00014 & $0.00048 \sqrt{9}$ & 0.00006 \\
\hline Cerebellar peduncle $†$ & 0.00006 & 0.00014 & 0.00014 \\
\hline Cerebellum $\dagger$ & 0.00006 & 0.00014 & 0.00014 \\
\hline
\end{tabular}

MS=Multiple sclerosis

${ }^{\star}$ Multiple comparison test.

†For the hemispheric regions, both right and left hemisphere values for MTR were included in the analysis.

All values indicate lower MTR in both MS groups $v$ control and lower MTR in familial MS patients $v$ sporadic MS patients with the exception,: $¥$ MTR higher in familial than in sporadic MS patients and $₫ M T R$ higher in sporadic MS patients than in controls. sporadic group ( $\mathrm{p}<0.05$; tables 3 and 4$)$. Particularly, significantly lower MTR were detected in patients (both groups) compared with the control in the centrum semiovale, corpus callosum (genu), frontal white matter, cerebellum, and cerebellar peduncles. Also the highest difference in MTR between familial and patients with sporadic MS was detected in the corpus callosum, cerebral peduncles, and cerebellar peduncles. There were no differences in mean MTR values between right and left hemispheric ROIs in all groups included in the study.

\section{Discussion}

Although conventional MRI showing focal lesions has been established as the most important paraclinical tool for diagnosis and monitoring efficacy of treatment in MS, necropsy studies indicated that the MS pathological process is not restricted to the focal lesions but is extended to the adjacent white matter. ${ }^{20-22}$ Most of these subtle microscopical lesions remain beyond the resolution of conventional MRI. ${ }^{33}$ To gain more data for total disease burden, also that beyond the resolution of conventional MRI, MTR has been applied; this provides information on the integrity of the macromolecular matrix and corellates with extension of pathological process in $M S .^{25}$ In the healthy population MTR values of the white matter was assessed to be between $32.0 \%$ and $50.8 \% .^{33-34}$ It has been shown that the MTR values for normal appearing white matter in patients with sporadic MS were decreased compared with the control group. ${ }^{34-36}$ Additionally, abnormal MTR values of normal appearing white matter were detected in patients with sporadic MS with negative conventional brain MRI. ${ }^{37}$ It has also been shown that there were no differences in mean MTR value between asymptomatic relatives of patients with sporadic MS and healthy persons. ${ }^{38}$ Astonishingly, there are no data on direct MTR comparison of normal appearing white matter between patients with familial MS and those with sporadic MS. We have conducted such a comparative MTR analysis, which aimed to contribute to the discussion on heterogeneity of MS and possible differentiation between familial and sporadic MS.

Although demographic and clinical data do not provide support for viewing familial MS as distinct from the sporadic form of disease, it is tempting to speculate that some peculiarities and differences still exist. In families heavily loaded with MS there was earlier onset of the disease and the male/female ratio was greater. ${ }^{17}$ Another argument that MS heterogeneity may have a genetic basis is relevant to an apparent difference between patients with MS in different parts of the world. ${ }^{39}$ Additionally, the rate at which disability develops seems to be a little more homogenous among patients with first degree relatives. ${ }^{18}{ }^{40}$ A higher proportion of patients with familial MS experienced a progressive clinical course of disease. Consistently with these findings, we have found lower MTR values for normal 
appearing white matter in patients with familial MS compared with patients with sporadic MS. We conclude that this difference is not caused by demographic or clinical features of the patient groups, as we have matched patients with familial MS and patients with sporadic MS for all variables which have been suggested to influence the MTR value. These variables included sex, age, time from the begining of disease, EDSS, type of disease, and total $\mathrm{T} 1$ and $\mathrm{T} 2$ lesion volume. ${ }^{32-34}$ There are no data on association between genetic polymorphism and familial MS but recently it has been suggested that genetic factors, Apo $\mathrm{E} 4$ isoform, ${ }^{41}$ interleukin $1 \mathrm{~b}$ and interleukin 1 receptor antagonist, ${ }^{42-43} \mathrm{Fc}$ gamma RIIIB, and Fc gamma RIIA ${ }^{44}$ are implicated in more progressive, severe forms of MS. Therefore, it might be tempting to speculate that in familial MS more widespread tissue destruction as detected by MTR might result from a genetic predisposition.

Apart from differences in mean MTR values between patients with familial MS and those with sporadic MS we have also found significant variations of MTR values in specific brain location (ROI analysis). In familial MS we found lower MTR values in most ROIs compared with the sporadic group but the most significant differences have occurred in the corpus callosum and in cerebral and cerebellar peduncles. This finding is of interest as focal MS lesions on MRI have also shown a tendency to occur in these locations with higher frequency. ${ }^{45}$ This would indicate that in familial MS the prelesion formation time is prolonged. Therefore, one possible explanation of our findings is that in familial patients reduction of MTR in normal appearing white matter might have preceded new lesion formation. ${ }^{46}$ Another possible explanation for such MTR ROI variation is that the nature of the changes in normal appearing white matter may be slightly different in familial and sporadic MS. Therefore, our results might indicate that in patients with familial MS the pathological processes spread more actively to the regions of highly myelinated white matter. This is in agreement with the results of a previous study indicating widespread white matter hypometabolism in patients with MS. ${ }^{47}$ Our results on the widespread decreases in MTR in white matter in patients with MS might explain functional deterioration by impairing axonal brain function and cortical and subcortical connectivity. The extent of decreases in MTR in patients with familial MS will have to be fully defined by MTR histogram analysis but our data indicate that widespread changes might be the MRI hallmark for familial MS.

The nature of discrete changes beyond the resolution of conventional MRI responsible for our MTR findings can only be speculative. However, the recent data on the correlation of decreased normal appearing white matter MTR values with brain atrophy suggests that tissue loss might be primarily responsible for the reduced value of MTR in MS brains. ${ }^{48}$ Axonal loss, either primary or secondary to focal abnormalities, resulting in wallerian degeneration should be considered in this regard. Postmortem study, both in humans and animals, showed a strong correlation between low MTR value and myelin and axonal loss. ${ }^{49}$ Additionally, in a recently published study myelin and axonal loss seemed to be the most likely contributors to the increased diffusivity in normal appearing white matter. ${ }^{50}$

In conclusion, our data suggest that there are differences in MTR analysis of normal appearing white matter in patients with familial MS and patients with sporadic MS. Patients with familial MS showed lower mean MTR values and more widespread MTR abnormalities. These findings might indicate slight differences in the extent and nature of white matter pathology in familial and sporadic MS.

This study was supported by a grant from KBN 4P05B12114 and the City Council of Lodz. We thank Dr Massimo Filippi, Neuroimaging Research Unit, Department of Neuroscience, Scientific Institute Ospedale San Raffaele, Milan, for helpful Scientific Institute Ospedale San Raffaele,
advice and critical discussion of our results.

1 Pratt RTC, Compston AD, McAlpine D. The familial incidence of disseminated sclerosis and its significance. Brain 1951;74:191-232.

2 Miller R. Genetic aspects of multiple sclerosis. Archives of Neurology and Psychiatry (Chicago) 1953;70: 733-40.

3 Shapira K, Poskanzer DC, Miller H. Familial and conjugal multiple sclerosis. Brain 1963; 86:315-32.

4 Mackay RP, Myrianthopoulos NC. Multiple sclerosis in twins and their relatives. Arch Neurol 1966;15:449-62.

5 Compston A. Genetic factors in the etiology of multiple sclerosis. In: McDonald WI, Silberg DH, eds. Multiple Sclerosis. London: Butterworths, 1986; 56-73.

6 Visscher BR, Detels R, Dudley J, et al. Genetic susceptibility Visscher BR, Detels R, Dudley J, et al. Genetic suscep
to multiple sclerosis. Neurology 1979;29:1354-60.

7 to multiple sclerosis. Neurology 1979;29:1354-60. saile RW, Hodge SE, Visscher BR, et al. Genetic
susceptility to multiple sclerosis: a linkage analysis with susceptibility to multiple sclerosis: a linkage analysis
age of oneset corrections. Clin Genet 1980;18:160-7.

8 Sadovnik AD, Baird PA. The familial nature of multiple Sadovnik AD, Baird PA. The familial nature of multiple
sclerosis: age-corrected empiric recurrence risk for children and siblings of patients. Neurology 1988;38:990-1.

9 Ebers GC, Sadovnik AD, Risch NJ and the Canadian Collaborative Study Group. A genetic basis for familial aggregation in multiple sclerosis. Nature 1995;377:150-1.

10 Sadovnik AD, Baird PA, Ward RH. Multiple sclerosis updated risk for relatives. Am $\mathcal{F}$ Med Genet 1988;29:53341.

11 Ebers GC, Bulman DE, Sadovnik AD, et al. A population based study of multiple sclerosis in twins. $N$ Engl f Med 1986;315:1638-42.

12 Mumford CJ, Wood NW, Kellar-Wood H, et al. The British Isles survey of multiple sclerosis in twins. Neurology 1994;44:11-15.

13 Sadovnick AD. Genetics of multiple sclerosis in British Columbia and throughout Canada. Ann Neurol 1994;36: S18-S21.

14 Oksenberg JR, Sebaun E, Hauser SL. Genetics of demyelinating disease. Brain Pathol 1996;6:289-302

15 Ebers GC, et al. A full genome search in multiple sclerosis. Nature Genetics 1996;13:472-6.

6 Dyment DA, Sadovnick AD, Ebers GC. Genetics of multiple sclerosis. Hum Mol Genet 1997;6:1693-98.

17 Ebers GC, Koopman WJ, Hader W, et al. The natural history of multiple sclerosis: a geographically based study 8: familial multiple sclerosis. Brain 2000;123:641-9.

18 Weinshenker BG, Bulman D, Carriere W, et al. A comparison of sporadic and familial multiple sclerosis. Neurology 1990;40:1354-8

19 McDonald WI. Diagnosis of multiple sclerosis. BMf 1989; 299:635-7.

20 Allen IV, McKeown SR. A histological, histochemical and biochemical study of the macroscopically normal white biochemical study of the macroscopically normal white
matter in multiple sclerosis. F Neurol Sci 1979;41:81-91.

21 Newcombe J, Cuzner ML, Roytta M, et al. White matter proteins in multiple sclerosis. F Neurochem 1980;34:700708.

22 Newcombe J, Woodroofe MN, Cuzner ML. Distribution of glial fibrillary acidic protein in gliosed human white matter.

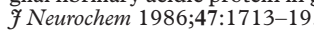

23 Lundbom N. Determination of magnetization transfer contrast in tissue: an MR imaging study of brain tumors. AfR Am F Roentgenol 1992;159:1279-85.

24 Wolff SD, Balaban RS. Magnetization transfer contrast (MTC) and tissue water proton relaxation in vivo. Magn Reson Med 1989;10:135-44.

25 Dousset V, Grossman RI, Ramer KN, et al. Experimental allergic encephalomyelitis and multiple sclerosis: lesion characterization with magnetization transfer imaging. Characterization with magnet

26 Lynch SG, Rose JW, Smoker W, et al. MRI in familial multiple sclerosis. Neurology 1990;40:900-3. 
27 Tienari PJ, Salonen O, Wilkstrom J, et al. Familial multiple sclerosis: MRI findings in clinically affected and unaffected sclerosis: MRI findings in clinically affected and unaffect
siblings. F Neurol Neurosurg Psychiatry 1992;55:883-6.

28 Fulton JC, Grossman RI, Mannon LJ, et al. Familial multiple sclerosis: volumetric assessment in clinically symptomatic and asymptomatic individuals. Mult Scler 1999;5 $74-77$

29 Poser CM, Paty DW, Schneiberg L, et al. New diagnostic criteria for multiple sclerosis: guidelines for research protocol. Ann Neurol 1983;13: 227-31.

30 Barkhof F, Filippi M, Miller DH, et al. Comparison of MRI critieria at first presentation to predict conversion to clinically definite multiple sclerosis. Brain 1997;120:2059-69.

31 Filippi M, Rocca MA, Comi G. Magnetization transfer ratio of multiple sclerosis lesions with variable duration of enhancement. F Neurol Sci 1998;159:162-5.

32 Brainin M, Neuhold A, Reisner T, et al. Changes within the "normal" cerebral white matter of multiple sclerosis patients during acute attacks and during high-dose patients during acute attacks and during high-dose Neurol Neurosurg Psychiatry 1989;52:1355-9.

33 Gass A, Barker GJ, Kidd D, et al. Correlation of magnetization transfer ratio with clinical disability in multiple sclerosis. Ann Neurol 1994;36:62-7.

34 Filippi M, Campi A, Dousset V, et al. A magnetization transfer imaging study of normal-appearing white matter in multiple sclerosis. Neurology 1995;45:478-82.

35 Loevner LA, Grossman RI, Cohen JA, et al. Microscopic disease in normal appearing white matter on conventional MR images in patients with multiple sclerosis: assessment with magnetization-transfer measurements. Radiology 1995; 196:511-55.

36 Hiehle JF, Lenkinski RE, Grossman RI, et al. Correlation of spectroscopy and magnetization transfer imaging in the evaluation of demyelinating lesions and normal appearing white matter in multiple sclerosis. Magn Reson Med 1994:32:285-93.

37 Filippi M, Rocca MA, Minicucci L, et al. Magnetization transfer imaging of patients with definite $\mathrm{MS}$ and negative transfer imaging of patients with definite MS
conventional MR. Neurology 1999;52:845-48.

38 Filippi M, Campi A, Martino G, et al. A magnetization transfer study of white matter in siblings of multiple sclerosis patients. $\mathscr{J}$ Neuro Sci 1997;147:151-3.
39 Hammond SR, McLeod JG, Macaskill P, et al. Multiple sclerosis in Australia: socio-economic factors. $\mathcal{F}$ Neurol sclerosis in Australia: socio-economic

40 Multiple Sclerosis Genetic Group. Clinical demographics of multiplex families with multiple sclerosis. Ann Neurol 1998;43:530-534.

41 Fazekas F, Strasser-Fuchs S, Schmidt H, et al. Apolipoprotein $\mathrm{E}$ genotype related differences in brain lesions of multiple sclerosis. 7 Neurol Neurosurg Psychiatry 2000;69:25-8.

42 Kantara $\mathrm{OH}$, Atkinson $\mathrm{E} J$, Hebrink DD, et al. Association of two variants in IL-1 $\beta$ and IL-1 receptor antagonist genes with multiple sclerosis $\mathcal{7}$ Neuroimmunol 2000;106:220-227.

43 Schrijver HM, Crusuis JBA, Uitehaag BMJ, et al. Association of interleukin-1 $\beta$ and interleukn 1-receptor antagonist genes with disease severity in MS. Neurolgy 1999;52: 595-9.

44 Myhr KM, Raknes G, Nyland H, et al. Immunoglobulin GFc-receptor ( $\mathrm{Fc} \gamma \mathrm{R})$ IIA and IIIB polymorphism related to disability in MS. Neurology 1999;52:1771-6.

45 Miller DH. Spectrum of abnormalities in multiple sclerosis. In: Miller DH, Kesselring J, McDonald WI, et al, eds. Magnetic Resonance in Multiple Sclerosis. Cambridge: Cambridge University Press, 1997;31-62.

46 Filippi M, Rocca M, Martino G, et al. Magnetization transfer changes in the normal appearing white matter precede the appearance of enhancing lesions in patients with multiple sclerosis. Ann Neurol 1998;512:1689-97.

47 Bakshi R, Miletich RS, Kinkel PR, et al. High-resolution fluorodeoxyglucose positron emission tomography shows both global and regional cerebral hypometabolism in multiple sclerosis. F Neuroimaging 1998;4:228-34.

48 Tortorella C, Viti B, Bozzali M, et al. A magnetization transfer histogram study of normal-appearing brain tissue in MS. Neurology 2000;54:186-93.

49 Van Waesberghe JHTM, Kamphorst W, De Groot CJA, et al. Axonal loss in multiple sclerosis lesions: magnetic resonance imaging insights into substrates of disability. Ann Neurol 1999;46:747-54.

50 Cercignani M, Iannucci G, Rocca MA, et al. Pathologic damage in MS assessed by diffusion-weighted and magnetization transfer MRI. Neurology 2000;54:1139-44. 